orbit force between a triton and a nucleus is the same as that between a nucleon and a nucleus.

Now the spin-orbit force is written as $V_{\mathrm{so}}(\mathrm{r}) \mathbf{L} \sigma$, where $\sigma$ is the spin of the projectile and $\mathbf{L}$ the relative angular momentum between the projectile and the target. For the same incident velocity this angular momentum is three times greater for a triton than for a nucleon, so we expect the spin-orbit potential depth to be one-third the nucleon value to leave the whole potential the same. Since the depth of the nucleon spin-orbit potential for nucleons is about $6 \mathrm{MeV}$ we expect a value of about $2 \mathrm{MeV}$ for the triton spin-orbit potential.

To test this prediction, the differential cross sections were first compared with calculations using a triton optical potential obtained by folding, and the parameters were slightly altered to optimise the fit. As shown in the figure, this gives a good fit to the cross section. The polarisations calculated with a spin-orbit potential of $2.5 \mathrm{MeV}$ are however far smaller than the measured values. Further calculations were made using optimum potentials obtained by Becchetti and Greenlees, with very similar results.

The parameters were then varied to try to fit the polarisations as well as the differential cross sections, and it was found necessary to alter the absorbing part of the potential as well as the spin-orbit potential itself. Potentials were found that fit the polarisations very well, but they have spin-orbit depths of $6 \mathrm{MeV}$, which is much greater than that given by the folding models.

The new data can therefore be

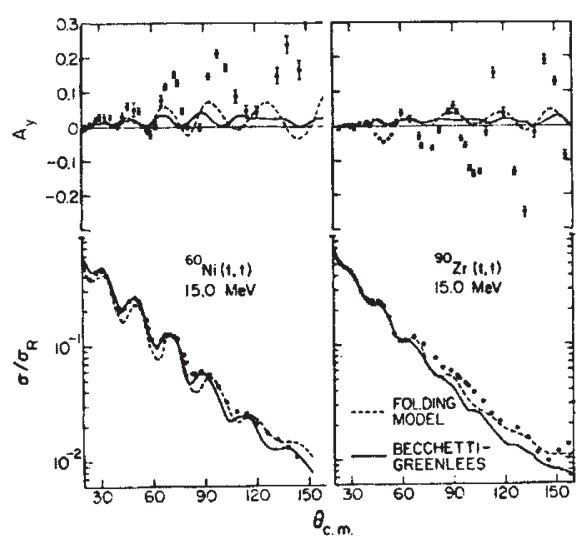

Polarisations (top) and differential cross-sections (bottom) for the elastic scattering of $15 \mathrm{MeV}$ tritons by ${ }^{60} \mathrm{Ni}$ and ${ }^{8} \mathrm{Zr}$ compared with optical model calculations with a folding model potential (dashed lines) and with a Becchetti-Greenlees potential (full lines). Both potentials fit the differential cross-sections quite well, but give polarisations that are far too small. successfully analysed by the optical model, but the strength of the spinorbit potential is unexpectedly high, and poses a challenging theoretical problem.

\section{Disturbed habitats}

\section{from Peter D. Moore}

ONE of the basic aims of ecological research is the generation of theoretical models on the basis of which predictions can be made. The complexity of interactions in any ecological system make this a difficult task, but it is a particularly necessary one especially where a basis is required for the formulation of an informed management programme for a given habitat. As a consequence of this need, there has been a noticeable swing in ecological research in recent years away from the traditional study of 'natural' ecosystems to an unashamed interest in those habitats which have been subjected to some disturbance or perturbation at the hands, or feet, of man.

A good example is that of sand dune habitats, where the bulk of ecological research interest is no longer focused on the general course of successional process, but has become concentrated on the effects of trampling upon soils and vegetation. Some recent work in this field by Liddle and Grieg-Smith (J. appl. Ecol., 12, 893 and 909; 1975) has involved both descriptive and experimental studies on the Abeffraw sand dunes in Anglesey.

Examination of soils revealed that several changes occur with trampling. Trampled soils were found to have increased in their bulk density and, as might be predicted, the effect of a car was considerably more severe than the passage of a human being, the density being increased by a factor of about $30 \%$ more after a car has passed than after a man. Soil compaction was associated with higher water content, which could favour certain species sensitive to water stress in this arid environment, but the anaerobic nature of such soils would not encourage extensive root growth by most species.

Examination of the vegetation from transects across paths showed that although species number decreased towards the path centre, diversity remained fairly constant. This suggests that loss in richness is accompanied by an increase in evenness of apportionment of biomass between species; this was probably caused by reduction in dominance with increased trampling pressure. Other observations show a fall in total biomass and an increase in the proportion of that biomass belonging to monocotyledenous species as trampling increases.

Unfortunately the vegetation data suffer from the disadvantage of having been gathered in June, when most of the winter annual species (mainly dicotyledons) would have already flowered and died. Since many of these species flourish under disturbed conditions, especially if sand is laid bare, they are likely to be an important winter and spring component of the path flora. Liddle and Grieg-Smith suggest that monocot: dicot ratio could prove a simple and useful index of trampling intensity, but seasonal factors need to be considered since there will be a greater proportion of dicots in winter.

Liddle (Biol. Conserv., 8, 251; 1975) has attempted to generalise yet further concerning the vulnerability of vegetation to trampling, on the basis of his sand dune data and further information derived from other habitats. He demonstrates a linear relationship between the logarithm of the number of people needed to pass along a linear path through a given vegetation type in order to reduce its biomass or cover by half, and the logarithm of the recorded primary productivity of that vegetation type. This relationship is not surprising, since more productive vegetation may be expected to regrow after trampling and so recover its former biomass more quickly. Liddle also considers that mature ecosystems are more resistant to trampling than early successional ones because they are more productive. This is not consistent with his own data, however, which show that short turf grassland containing such species as Festuca rubra, Bellis perennis, and Poa pratensis are particularly resistant to trampling yet are not mature in the successional sense, unless one regards the path community as a 'trampling climax', in which case the argument becomes a semantic one. The productivity/trampling resistance relationship may be genuine, but there is a danger of confusing net and gross ecosystem production when making successional inferences. Net ecosystem production does not rise during the early stages of succession, but then falls to zero at climax. Some of the most productive plant species are in fact found in immature ecosystems. It is therefore unwise to state that mature ecosystems are more productive, and therefore, more resistant to trampling.

The development of predictive models, or even the derivation of simple indices for the monitoring of man-induced changes is still some way off. But if Liddle's ideas on productivity are sufficiently compacted to hold water then we should be able to increase an ecosystem's tolerance to trampling simply by fertilising it. 